\title{
Influencing Factors of Outpatient Medication Costs for Chronic Diseases in China from 2015 to 2018: A Retrospective Study
}

\author{
Jiajia Xie ${ }^{1}$, Yan Qian ${ }^{2}$, Yi Wang ${ }^{1}$, Xun Liu ${ }^{1}$ and Dan Deng ${ }^{1, *}$ \\ ${ }^{1}$ School of Public Health and Management, Chongqing Medical University. Research Center for Medicine and Social Development. Innovation \\ Center for Social Risk Governance in Health, China \\ ${ }^{2}$ Department of Pharmacy, the Second Affiliated Hospital of Chongqing Medical University, China
}

*Corresponding author: Dan Deng, Department of Health Statistics, School of Public Health and Management, Chongqing Medical University, 1 Yixueyuan Road, Yuzhong District, Chongqing 400016, China, E-mail: 100079@cqmu.edu.cn

Received: 02 Dec, 2019 | Accepted: 24 Dec, 2019 | Published: 31 Dec, 2019

Citation: Xie J, Qian Y, Wang Y, Liu X, Deng D (2019) Influencing Factors of Outpatient Medication Costs for Chronic Diseases in China from 2015 to 2018: A Retrospective Study. J Epidemiol Public Health Rev 4(3): dx.doi.org/10.16966/2471-8211.179

Copyright: (C) 2019 Xie J, et al. This is an open-access article distributed under the terms of the Creative Commons Attribution License, which permits unrestricted use, distribution, and reproduction in any medium, provided the original author and source are credited.

\begin{abstract}
Background: Chronic diseases have always been a global public health issue. The costs of lifelong chronic disease treatment have resulted in heavy economic burdens to patients and society. The purpose of this study is to explore the influencing factors and current situation of drug expenditures in chronic disease outpatient clinics to provide a reference for alleviating the economic burden and preventing and managing chronic diseases efficiently.
\end{abstract}

Methods: A retrospective analysis of outpatient drug expenditure data for diabetes mellitus, hypertension and coronary heart disease from January 2015 to December 2018 in a tertiary first-class hospital in Chongqing was performed. The data were obtained from outpatient drug cost records. Univariate analysis was used to analyse the differences among the different drug cost groups. Logistic regression was used to explore the influencing factors of outpatient drug costs. Forest plots were used to visualize the results of the influencing factors of drug costs for the different chronic diseases.

Results: From 2015 to 2018, there were 190474 diabetes mellitus patients, 129535 hypertension patients, and 21982 coronary heart disease patients. The drug cost per capita for coronary heart disease patients was the highest ( 687.53 Yuan), followed by diabetes mellitus (582.50 Yuan) and hypertension (473.30 Yuan). The influencing factors included sex (except for hypertension), age, medical payment method, category of major drug, doctor's title, department, and full implementation of the zero mark-up policy.

Conclusions: Long-term medication for chronic diseases is expensive; thus, it is necessary to control the cost of medication. The implementation of the zero mark-up policy has effectively controlled drug costs for patients with chronic diseases. The effect of the different medical payment methods on outpatient drug expenses is also obvious. Reducing or controlling high outpatient drug costs is a complex and long-term process that requires multi-sectoral and multi-faceted coordination and cooperation.

Keywords: Chronic diseases; Medication cost; Influence factor; Outpatient department

\section{Introduction}

Chronic Non-Communicable Diseases (NCDs), as well as chronic diseases, have become increasingly serious and are a major global public health concern [1]. Chronic diseases have long incubation periods, long disease courses and complicated aetiologies; they are caused by internal and external factors [2]. In recent years, the prevalence of chronic diseases has increased rapidly, with an annual increasing trend in young populations, resulting in significant medical and health challenges $[3,4]$. According to World Health Organization (WHO) statistics in 2018, chronic diseases were responsible for 41 million deaths annually, accounting for $71 \%$ of all deaths globally; cardiovascular diseases accounted for the majority (approximately $44 \%$ ) of chronic disease deaths [5]. As a populous country, China reports an astonishing number of deaths from chronic diseases. A total of $89.3 \%$ of all deaths are attributed to chronic diseases, which are the main threats to Chinese health [6].

Chronic diseases not only result in heavy disease burdens for patients but also result in substantial economic cost burdens for families and society, either directly or indirectly [7-9]. Because chronic diseases require lifelong medication and treatment, they are more likely to involve long-term and sustained medical expenses; an inability to pay may cause additional illness in many families. Many studies have shown that chronic diseases increase the family cost of health care, which leads to higher economic cost risks for families with chronic diseases than for healthy families [10]. This contradiction is especially prominent in developing countries. NCDs not only result 
in economic burdens for families but also increase social and national financial pressure. As chronic diseases become increasingly common, the people's demand for health care services will exceed the supply, and the cost of health care will gradually increase. Understanding the causes of the expansion of medical costs and preventing an excessive increase in medical costs are very important for the development of health care for individuals, families and countries. The Chinese government has also adopted a series of medical reform measures to solve the problem of increasing medical expenses for NCDs. In 2009, China launched a series of large and complex new health reforms. The main objectives of health care reform are to reform health care financing, basic drug policies and public hospitals to provide affordable and equitable basic health care for all $[11,12]$. It has been 10 years since the implementation of the new medical reform, which has exerted a tremendous influence on the development of Chinese health care. The reform focuses on urban and rural residents and includes medical insurance, medicines, public health services, hospitals, health care and other aspects. Within the reform, the three pillars of medical insurance, drug pricing and public hospitals have become the main focus of the reform [13]. As one of the key points of reform, China's medical insurance plays an important role in reducing economic burden for patients. Since the implementation of China's basic medical insurance system, there has been a medical insurance system covering Urban Employee Medical Insurance (UEMI), Urban Resident Medical Insurance (URMI) and New Rural Cooperative Medical Scheme (NRCMS) as the main body. Combined together, the basic health insurance covers $95 \%$ of the total population [14]. China uses a combination of government-guided prices and market pricing in the pricing of drug prices. According to the system, drugs can be divided into two categories: medical insurance drugs and non-medical insurance drugs. Medical insurance drugs are divided into two categories: A and B, with different price management. Due to the information asymmetry in the pharmaceutical market, there are many kinds of pharmaceutical market, which results in the difference of pharmaceutical cost and the complexity of the composition of pharmaceutical cost $[15,16]$. In order to better control the cost of drugs, since 2017, China has completely cancelled the drug addition fee (except for Chinese herbal pieces), and implemented zero price difference for drugs. Specifically, it refers to the sale of drugs by medical institutions or pharmacies to patients at the purchase price.

Hospital outpatient services, as an effective external business practice of hospitals and the first contact for diagnosis and treatment, can reflect the situation of patients with chronic diseases and the medical costs borne by patients to a certain extent. Patients with chronic diseases need long-term medication rather than hospitalization, and active and sustained drug treatment can effectively prevent the deterioration of chronic diseases and the occurrence of serious complications. The long-term therapeutic treatment of patients with chronic diseases is mainly implemented through prescription drugs in hospital outpatient clinics [17]. In addition, patients can choose doctors based on their financial situation and illness, which is also related to costs. Therefore, the primary medical expenditures of patients with chronic diseases are outpatient drug expenditures. In China, most of the studies on the medical expenses associated with chronic diseases focus on hospitalization and hospitalization nursing expenses [12,18]. For outpatient related research, more research is focused on outpatient prediction, and there are few studies on basic outpatient medical expenses [19-21].

Thus, the purpose of this study is to explore the influencing factors of outpatient drug expenditures for chronic diseases in a third-class a hospital in Chongqing, China. Chinese medical insurance system and zero mark-up policy were combined to analyse the implementation of the medical and health system reform policy. These results can provide a reference for improving medical and health management, guiding doctors on the rational use of drugs, reducing the economic burden of chronic diseases and preventing and managing chronic diseases.

\section{Methods \\ Data resources}

The data were collected from drug expense data recorded in the Hospital Information System (HIS) of an outpatient clinic of a thirdlevel hospital in Chongqing, China, from January 2015 to December 2018. According to the ranking of outpatient diagnostic codes, the data of the top three chronic diseases (diabetes mellitus, hypertension, and coronary atherosclerotic heart disease) were extracted. The name of diagnosis was single disease diagnosis, excluding two or more diagnoses. The extracted data were that the diagnosis names were all single disease, without complications. The dataset were covered case number, sex (female and male), age, diagnostic name, prescription date, medical payment method Out-of-pocket (OOP), Urban Employee Medical Insurance (UEMI), Urban Resident Medical Insurance (URMI), and Retired Cadre Medical Insurance (RCMI), category of major drug (western medicine and non-western medicine), doctor's title (primary, intermediate, deputy senior, senior and other), department (endocrinology and metabolism, general internal medicine, TCM-WM, cardiovascular, Geriatrics and other), full implementation of the zero mark-up policy (according to the full implementation of zero drug price difference in Chongqing on September 9, 2017, it is divided into pre policy and post policy) and outpatient drug expenses. The total number of diabetes mellitus patients was 190474 , the total number of hypertension patients was 129535 , and the total number of coronary heart disease patients was 21982.

\section{Research methods}

Chongqing has fully implemented the zero mark-up policy since September 9, 2017. An exploratory data analysis was conducted for patient sex, age, medical payment method, category of major drug, doctor's title, department, full implementation of the zero mark-up policy, and outpatient drug expenses. The outpatient drug expenses were regarded as the dependent variable, and the other variables were considered independent variables for the descriptive, univariate and multivariate logistic regression analyses (Table 1).

\section{Statistical analysis}

A descriptive analysis and chi-square analysis were performed using SPSS 22.0. A multivariate logistic regression analysis was performed using SAS9.4. The mean \pm standard deviation was used for the descriptive statistics. The non-normally distributed variables were represented by the median $\left(25^{\text {th }}\right.$ percentile, $75^{\text {th }}$ percentile). Count data were described by the frequency or constituent ratio. A chi-square test was used to determine the factors related to drug costs in outpatient clinics for chronic diseases. Multivariate logistic regression was used to explore the probable influencing factors of outpatient drug expenses. The significance threshold was $\mathrm{P}<0.05$. Finally, the forest plots were constructed with R3.5.3.

\section{Results \\ Basic information}

From 2015 to 2018, 341991 patients, including 171594 males and 170397 females, with diabetes mellitus, hypertension and coronary heart disease visited an outpatient clinic of a third-class a hospital in 
Table 1: Variable and variable assignment.

\begin{tabular}{|c|c|c|c|}
\hline Variable type & Variable & Detailed description & Value range \\
\hline Dependent variable & Outpatient drug expenses & Qualitative variable (2 level) & $\begin{array}{l}0=\text { low }(\leq \text { median }) \\
1=\text { high }(>\text { median })\end{array}$ \\
\hline \multirow[t]{7}{*}{ Independent variable } & Sex & Qualitative variable (2 level) & $\begin{array}{l}0=\text { female } \\
1=\text { male }\end{array}$ \\
\hline & Age & Qualitative variable (3 level) & $\begin{array}{l}1=(0 \sim 45 \text { years old }) \\
2=(45 \sim 65 \text { years old }) \\
3=(>65 \text { years old })\end{array}$ \\
\hline & Medical payment method & Qualitative variable (4 level) & $\begin{array}{l}\text { 1=Out-of-pocket (OOP) } \\
\text { 2=Urban Employee Medical Insurance (UEMI) } \\
\text { 3=Urban Resident Medical Insurance (URMI) } \\
\text { 4=Retired Cadre Medical Insurance (RCMI) }\end{array}$ \\
\hline & Category of major drug & Qualitative variable (2 level) & $\begin{array}{l}0=\text { Non-Western Medicine } \\
1=\text { Western Medicine }\end{array}$ \\
\hline & Doctor's title & Qualitative variable (5 level) & $\begin{array}{l}\text { 1=Primary } \\
\text { 2=Intermediate } \\
\text { 3=Deputy senior } \\
\text { 4=Senior } \\
\text { 5=Other }\end{array}$ \\
\hline & Department & Qualitative variable (6 level) & $\begin{array}{l}\text { 1=Other } \\
\text { 2=Endocrinology and metabolism } \\
3=\text { General internal medicine } \\
\text { 4=TCM-WM } \\
\text { 5=Cardiovascular } \\
6=\text { Geriatrics }\end{array}$ \\
\hline & $\begin{array}{l}\text { Full implementation of the zero } \\
\text { mark-up policy }\end{array}$ & Qualitative variable (2 level) & $\begin{array}{l}0=\text { Before } \\
1=\text { After }\end{array}$ \\
\hline
\end{tabular}

Chongqing. The total number of diabetes mellitus patients was 190474; 129535 patients were hypertension patients and 21982 patients were coronary heart disease patients. The outpatient drug expenses per person for diabetes, hypertension and coronary heart disease were $582.50(340.76,973.53)$ Yuan, $473.30(252.18,804.68)$ Yuan and 687.53 $(404.76,1001.62)$ Yuan, respectively. The patients whose outpatient drug costs were higher than median cost were considered the highcost group, while the remaining patients were considered low-cost group (including the median). The average ages of the diabetes mellitus, hypertension, and coronary heart disease patients were 63, 65 and 68 years old, respectively. The areas under the receiver operating characteristic (ROC) curve were $0.63,0.69$ and 0.68 respectively.

\section{Univariate analysis}

The results of the univariate analysis showed that sex (excluding hypertension), age, medical payment method, category of major drug, doctor's title, department, and full implementation of the zero markup policy were all related to the expenses of drugs in outpatients with the three studied chronic diseases.

From table 2, in terms of sex, there was a significant difference in the outpatient drug costs for diabetes and coronary heart disease $(\mathrm{P}<0.05)$, the difference was between low and high cost patient groups. But there was no difference in the outpatient costs for hypertension $(\mathrm{P}>0.05)$. The patients with chronic diseases were mainly over 45 years old. The number of coronary heart disease and hypertension patients over 65 years old who experienced high costs was higher than that of patients under 65 years.

Among the out-of-pocket (OOP), Urban Employee Medical Insurance (UEMI), Urban Resident Medical Insurance (URMI) and Retired Cadre Medical Insurance (RCMI) medical payment methods, we found that the chi-square values of diabetes mellitus, hypertension and coronary heart disease were $6574.118,3513.837$ and 335.668, respectively. The $\mathrm{P}$ values were all $\mathrm{P}<0.001$. There was a significant difference between these 2 groups. The difference was between low and high cost patient groups. More than $85 \%$ were UEMI patients, and less than 1\% were RCMI patients, but the cost associated with retired patients was much higher than the costs associated with the other medical payment methods. The costs of medicine for patients with coronary heart disease was as high as $1090.24(440.17,1411.67)$ Yuan, for patients with diabetes was as high as $792.27(364.94,407.82)$ Yuan, and for patients with hypertension was as high as 575.22 (216.99, 950.34) Yuan (Figure 1).

There were differences in outpatient drug expenses between different doctor titles and departments $(\mathrm{P}<0.05)$. The difference was between low and high cost patient groups. According to the doctor's title, there were significant differences in the outpatient drug costs between the high-cost and low-cost groups for diabetes mellitus $(\mathrm{P}<0.001)$, hypertension $(\mathrm{P}<0.001)$, and coronary heart disease $(\mathrm{P}<0.001)$. From the perspective of departments, the outpatient drug costs for diabetes $(\mathrm{P}<0.001)$, hypertension $(\mathrm{P}<0.001)$, and coronary heart disease $(\mathrm{P}<0.001)$ were significantly different. The difference was between low and high cost patient groups. When patients with chronic diseases selected outpatient doctors, they mainly selected a title above deputy senior, accounting for more than $50 \%$. The main chronic disease outpatient departments were the endocrinology and metabolism, general internal medicine, traditional Chinese medicineWestern medicine (TCM-WM), cardiovascular and geriatrics departments (Figures 2 and 3).

The analysis showed that since fully implementing the zero markup policy in Chongqing on September 9, 2017, the cost of drugs in 
Table 2: Univariate analysis of sex and age.

\begin{tabular}{|c|c|c|c|c|c|c|}
\hline \multirow[b]{2}{*}{ Variable } & \multicolumn{2}{|c|}{ Diabetes mellitus } & \multicolumn{2}{|c|}{ Hypertension } & \multicolumn{2}{|c|}{ Coronary heart disease } \\
\hline & $\begin{array}{l}\text { Low outpatient } \\
\text { drug expenses } \\
\text { (N=95239) } \\
N(\%)\end{array}$ & $\begin{array}{l}\text { High outpatient } \\
\text { drug expenses } \\
\text { (N=95235) } \\
N(\%)\end{array}$ & $\begin{array}{l}\text { Low outpatient } \\
\text { drug expenses } \\
\text { (N=64772) } \\
N(\%)\end{array}$ & $\begin{array}{l}\text { High outpatient } \\
\text { drug expenses } \\
(\mathrm{N}=64763) \\
\mathrm{N}(\%)\end{array}$ & $\begin{array}{l}\text { Low outpatient drug } \\
\text { expenses } \\
(\mathrm{N}=10991) \\
N(\%)\end{array}$ & $\begin{array}{l}\text { High outpatient } \\
\text { drug expenses } \\
\text { (N=10991) } \\
N(\%)\end{array}$ \\
\hline Sex & - & - & - & - & - & - \\
\hline Female & $45257(47.5)$ & $47677(50.1)$ & $34290(52.9)$ & $34589(53.4)$ & 4595(41.8) & $3989(36.3)$ \\
\hline Male & $49982(52.5)$ & $47558(49.9)$ & $30482(47.1)$ & $30174(46.6)$ & $6396(58.2)$ & 7002(63.7) \\
\hline$P$ value & \multicolumn{2}{|c|}{$<0.001$} & \multicolumn{2}{|c|}{0.091} & \multicolumn{2}{|c|}{$<0.001$} \\
\hline Age & - & - & - & - & - & - \\
\hline 0 45 years old & $9758(10.2)$ & $5112(5.4)$ & 6956(10.7) & 1984(3.1) & $381(3.5)$ & 205(1.9) \\
\hline 45 65 years old & $51691(54.3)$ & $46718(49.0)$ & $32563(50.3)$ & $25203(38.9)$ & $4702(42.8)$ & 4359(39.7) \\
\hline$>65$ years old & $33790(35.5)$ & $43405(45.6)$ & $25253(39.0)$ & $37576(58.0)$ & 5908(53.7) & $6427(58.4)$ \\
\hline$P$ value & \multicolumn{2}{|c|}{$<0.001$} & \multicolumn{2}{|c|}{$<0.001$} & \multicolumn{2}{|c|}{$<0.001$} \\
\hline $\begin{array}{l}\text { Category of major } \\
\text { drug }\end{array}$ & - & - & - & - & - & - \\
\hline $\begin{array}{l}\text { Non-Western } \\
\text { Medicine }\end{array}$ & $2640(2.8)$ & $171(0.2)$ & $4057(6.3)$ & $580(0.9)$ & 1284(11.7) & $67(0.6)$ \\
\hline Western Medicine & 92599(97.2) & $95064(99.8)$ & $60715(93.7)$ & 64183(99.1) & $9707(88.3)$ & 10924(99.4) \\
\hline$P$ value & \multicolumn{2}{|c|}{$<0.001$} & \multicolumn{2}{|c|}{$<0.001$} & \multicolumn{2}{|c|}{$<0.001$} \\
\hline
\end{tabular}

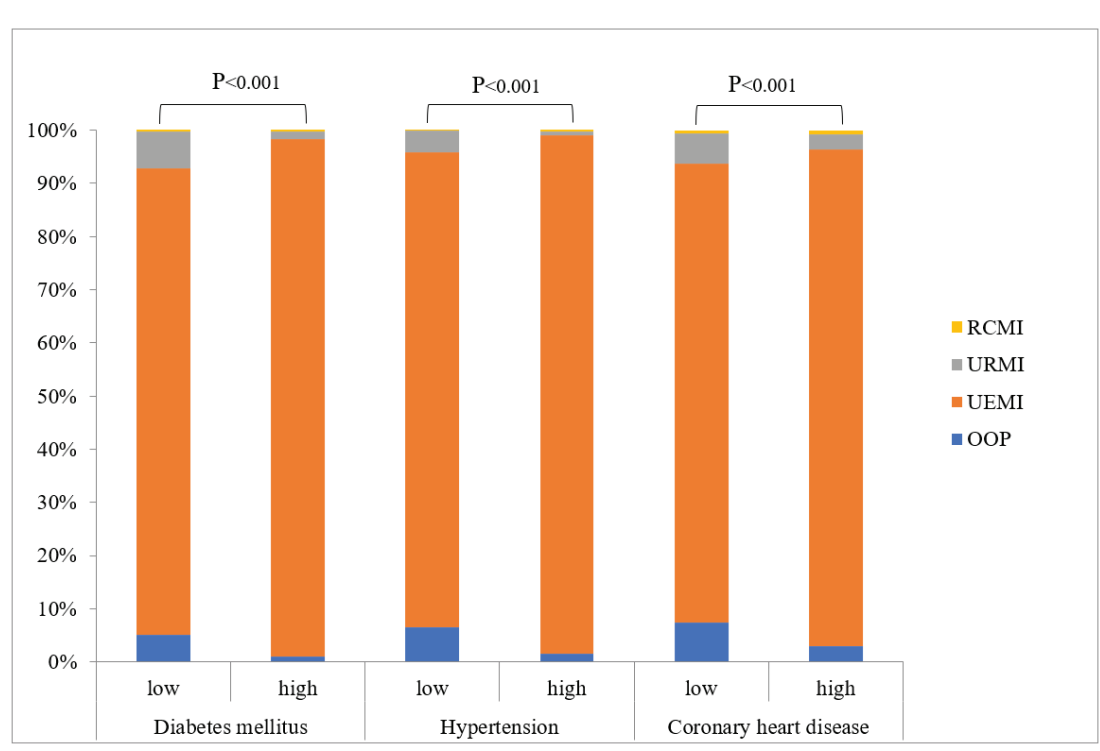

Figure 1: Univariate analysis of medical payment method.

different outpatient clinics has varied. There were significant differences in outpatient drug costs before and after the zero markup policy for diabetes mellitus $(\mathrm{P}<0.001)$, hypertension $(\mathrm{P}<0.001)$, and coronary heart disease $(\mathrm{P}<0.001)$. The difference was between low and high cost patient groups. The cost of diabetes mellitus drugs decreased from $604.65(344.90,1012.63)$ Yuan to 548.35(317.29, 901.15) Yuan, the cost of hypertension drugs decreased from 484.79 $(253.60,825.50)$ Yuan to $435.78(248.22,735.63)$ Yuan, the cost of coronary heart disease drugs decreased from $714.16(420.06,1028.20)$ Yuan to $621.02(369.58,935.51)$ Yuan, and the cost of drugs for outpatient service decreased (Figure 4).

\section{Influencing factors of outpatient drug expenses}

Seven independent variables were introduced into the logistic regression analyses of diabetes mellitus, coronary heart disease and hypertension, then the stepwise regression analysis model of hypertension excluded 1 insignificant variable: 'sex'. The results showed that the regression models of diabetes mellitus, hypertension and coronary heart disease were significant $(\mathrm{P}<0.001)$. Diabetes mellitus patients were concentrated in the department of endocrine and metabolic diseases, while hypertension and coronary heart disease patients were mainly concentrated in the department of cardiovascular medicine. 


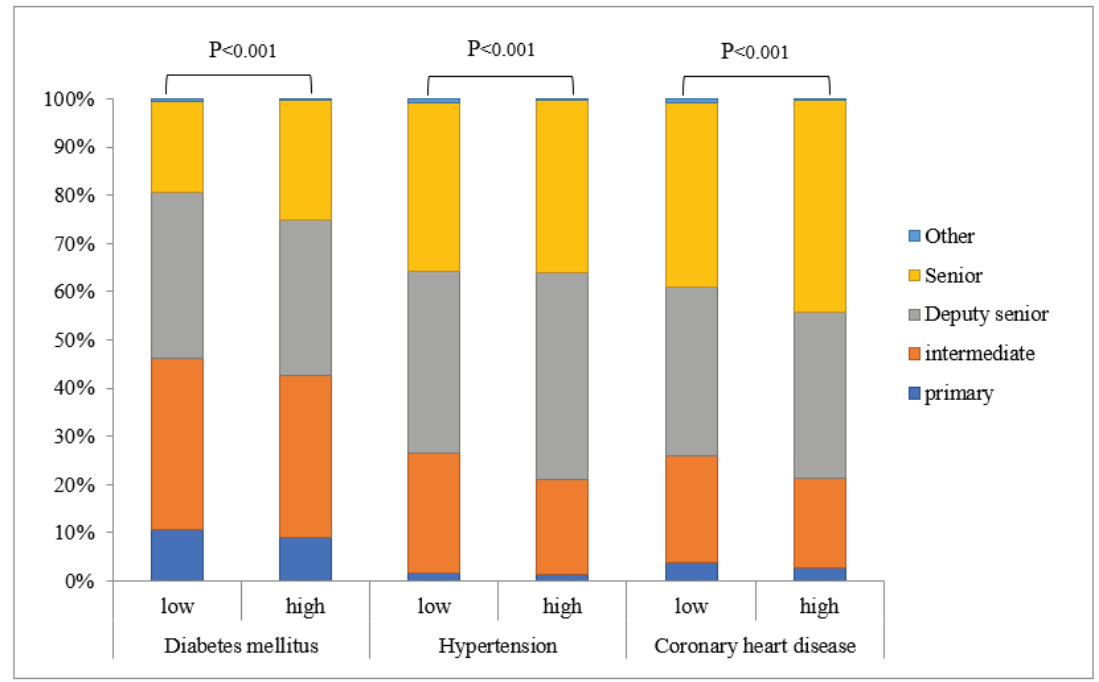

Figure 2: Univariate analysis of doctors' professional titles.

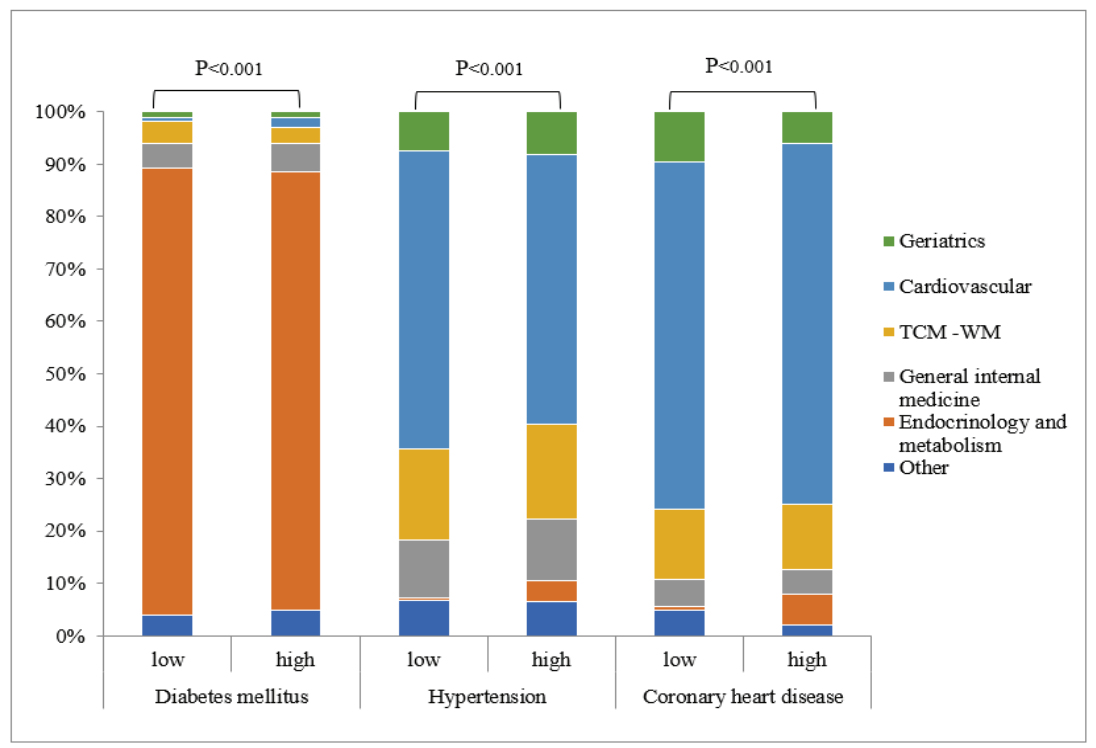

Figure 3: Univariate analysis of departments.

For diabetes mellitus, the control factors of outpatient drug cost were male, non-western medicine and full implementation of the zero mark-up policy. Among the multi-categorical variables, being 45-65 years old and over 65 years old were outpatient drug expenses inflation factors (OR: 1.493, 95\% CI: 1.438-1.550, P<0.0001; OR: 2.063, 95\% CI: 1.985-2.144, $\mathrm{P}<0.0001$, respectively). UEMI and RCMI, but not OOP, were outpatient drug expenditure inflation factors (OR: 5.400, 95\% CI: 5.036-5.789, P<0.0001; OR: 5.835, 95\% CI: 4.717-7.219, $\mathrm{P}<0.0001$, respectively). Intermediate, deputy senior and senior, but not primary, titles were outpatient drug costs inflation factors (OR: 1.057, 95\% CI: 1.021-1.194, P=0.0016; OR: 1.046, 95\% CI: 1.011-1.084, P=0.0109; OR: 1.441, 95\% CI: $1.389-$ $1.495, \mathrm{P}<0.0001$, respectively) (Figure 5).
For hypertension, non-Western medicine and full implementation of the zero mark-up policy were control factors for outpatient medical costs. The risk of high outpatient drug cost of Western medicine was 10.118 times higher than that of non-Western medicine. There was a significant difference before and after the full implementation of the zero mark-up policy (OR: 0.753, 95\% CI: 0.732-0.774, $\mathrm{P}<0.0001$ ). Age (reference: $0 \sim 45$ years old, $45 \sim 65$ years old OR: $2.434,95 \%$ CI: $2.305-2.569, \mathrm{P}<0.0001$; $>65$ years old OR: 4.840 , 95\% CI: $4.584-$ 5.110, $\mathrm{P}<0.0001$ ), medical payment method (reference: OOP, UEMI OR: 3.770, 95\% CI: 3.509-4.050, P<0.0001;RCMI OR: 3.064, 95\% CI: 2.324-4.041, $\mathrm{P}<0.0001$ ) and doctor's title (reference: primary, deputy senior OR: $1.526,95 \%$ CI: $1.380-1.687, \mathrm{P}<0.0001$; senior OR: 1.510 , 95\% CI: 1.364-1.672, $\mathrm{P}<0.0001)$ influenced outpatient drug expenses for chronic diseases (Figure 6). 


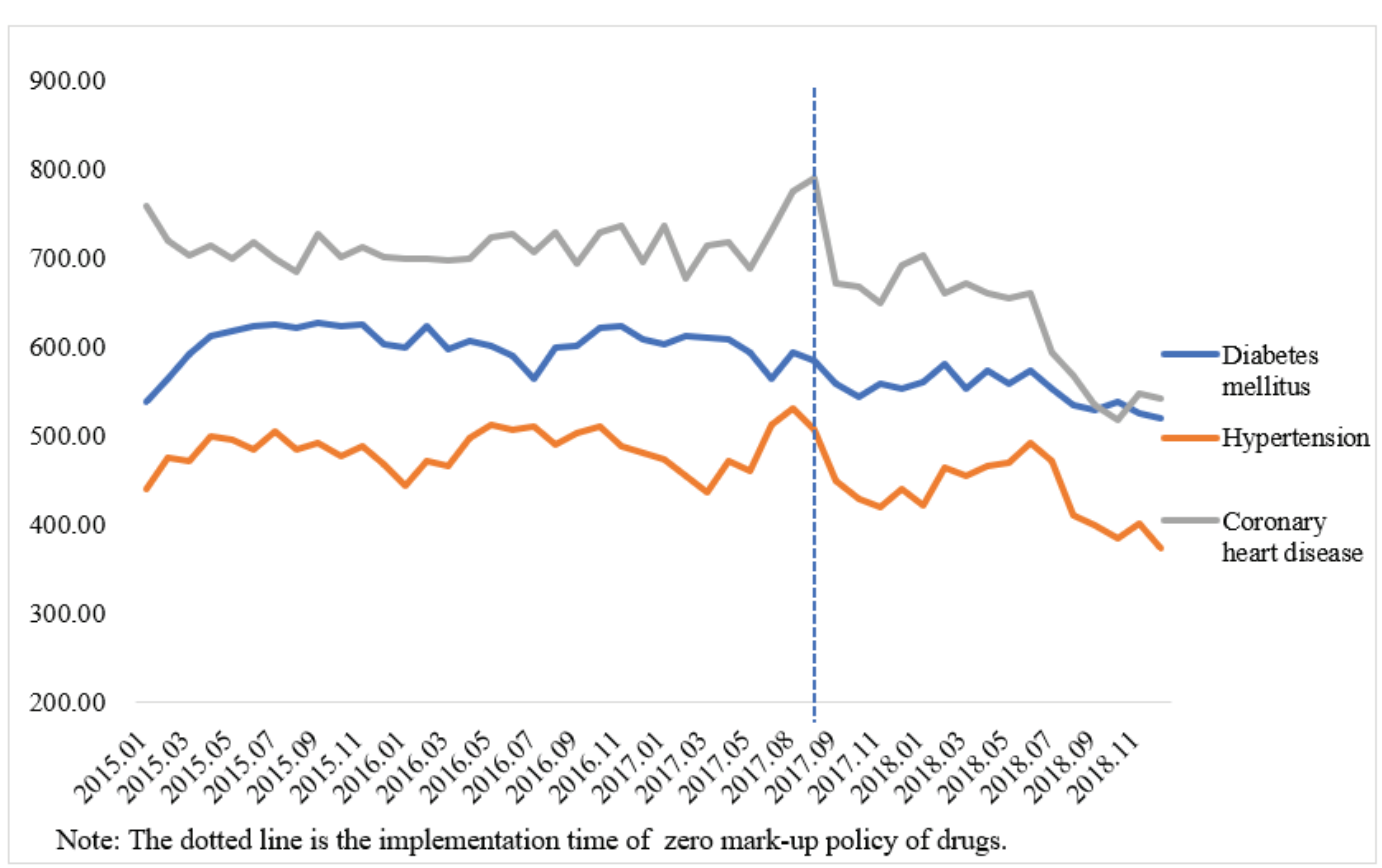

Figure 4: Time trend map before and after full implementation of the zero mark-up policy.

Variable
Sex
female
male
Age
$0 \sim 45$ years old
$45 \sim 65$ years old
>65 years old
Medical payment method
OOP
UEMl
RCMl
Category of major drug
Non-Western Medicine
Western Medicine
Doctor's title
primary
intermediate
Deputy senior
Senior
Department
Other
Endocrinology and metabolism
General internal medicine
TCM -WM
Cardiovascular
Geriatrics
Full implementation of the zero mark-up policy
Before
After

n

92934

97540

14870

98409

77195

5830

176127

409

2811

187663

18853

65849

63435

41511

8445

160946

9539

6909

2476

2159

126979

63495

\section{OR $(95 \% \mathrm{CI})$}

$0.909(0.892-0.926)$

$1.493(1.438-1.550)$

$2.063(1.985-2.144)$

$5.400(5.036-5.789)$

$5.835(4.717-7.219)$

$19.952(17.011-23.401)$

$1.057(1.021-1.194)$

$1.046(1.011-1.084)$

$1.441(1.389-1.495)$

$0.728(0.695-0.763)$

$0.831(0.781-0.884)$

$0.903(0.840-0.970)$

$1.668(1.507-1.847)$

$0.593(0.538-0.654)$

$0.797(0.781-0.814)$
$<0.0001$

$<0.0001$

$<0.0001$

$<0.0001$

$<0.0001$

$<0.0001$

0.0016

0.0109

$<0.0001$

$<0.0001$

$<0.0001$

0.0053

$<0.0001$

$<0.0001$

$<0.0001$

Figure 5: Forest plot for diabetes mellitus. 


Variable
Age
$0 \sim 45$ years old
$45 \sim 65$ years old
$>65$ years old
Medical payment method
OOP
UEMl
URMl
RCMl
Category of major drug
Non-Western Medicine
Western Medicine
Doctor's title
primary
Deputy senior
Senior
Department
Other
Endocrinology and metabolism
General internal medicine
TCM -WM
Cardiovascular
Geriatrics
Full implementation of the zero mark-up policy
Before
After

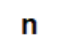

8940

57766

62829

5243

120882

3178

232

4637

124898

1965

52319

45745

8538

2931

14781

23040

70026

10219

99728

29807
OR(95\% $\mathrm{Cl})$

$<0.0001$

$<0.0001$

$<0.0001$

0.0064

$<0.0001$

$<0.0001$

$<0.0001$

$<0.0001$

$<0.0001$

$<0.0001$

$<0.0001$

$<0.0001$

$<0.0001$ $<0.0001$

Figure 6: Forest map of hypertensive.

In the analysis of coronary heart disease drug expenses, women, non-Western medicine and full implementation of the zero mark-up policy were outpatient drug cost reduction factors. Females spent less on outpatient drug expenses than males (OR: 1.219, 95\% CI: 1.149-1.299, $\mathrm{P}<0.0001)$. The risk of inflation of outpatient drug cost for non-Western medicine was only 0.038 times higher than that of Western medicine. The risk of inflation associated with the full implementation of the zero mark-up policy was 0.66 times higher than that associated with non-implementation. Being 4565 years old and over 65 years old were outpatient drug expense inflation factors(reference: $0 \sim 45$ years old, $45 \sim 65$ years old OR: 1.466, 95\% CI:1.220-1.763, $\mathrm{P}<0.0001$; $>65$ years old OR: $1.848,95 \%$ CI:1.538-2.219, $\mathrm{P}<0.0001)$. Patients who paid UEMI and RCMI spent higher than outpatients without medical insurance (reference: OOP, UEMI OR: 2.827, 95\% CI: 2.466-3.241, P<0.0001; URMI OR: 1.396, 95\% CI: 1.150-1.694, P=0.0007; RCMI OR: 4.007, 95\% CI: 2.760-5.819, $\mathrm{P}<0.0001$ ) (Figure 7).

\section{Discussion}

As the population ages, the prevalence of chronic diseases will continue to increase $[22,23]$. Once an individual develops a disease, the normal life of the patient will be affected. Long-term and frequent outpatient treatment and medication expenses are the main causes of poverty. According to the results, the expenses of patients with chronic diseases such as diabetes, hypertension, and coronary heart disease increased with age, especially in the middle-aged and elderly populations over 45 years old, which was an inflation factor of outpatient drug expenses. The rate of high outpatient drug cost of diabetes patients aged 45-65 was 1.493 times higher than that of patients under 45 years old (OR: 1.493, 95\% CI: 1.438-1.550, $\mathrm{P}<0.0001$ ); the odds ratio of patients over 65 years old was 2.063 times higher than that of patients under 45 years old (OR: 2.063, 95\% CI: $1.985-2.144, \mathrm{P}<0.0001)$. The rate of high outpatient drug cost of hypertension aged 45-65 was 2.434 times higher than that of patients under 45 years old (OR: 2.434, 95\% CI: $2.305-2.569, \mathrm{P}<0.0001$ ); the odds ratio of patients over 65 years old was 2.063 times higher than that of patients under 45 years old (OR: $4.840,95 \%$ CI: 4.584-5.110, $\mathrm{P}<0.0001)$; patients with coronary heart disease aged $45-65$ years and over were 1.466 and 1.848 times the risk of high outpatient drug costs than those aged 0-45(OR: 1.466, 95\% CI: 1.220-1.763, P<0.0001; OR: $1.848,95 \%$ CI: $1.538-2.219, \mathrm{P}<0.0001)$. The elderly patients not only had an increased number of outpatient visits but also had increased outpatient drug costs, consistent with $\mathrm{Lu} \mathrm{WH}$, et al. [24] and Wang $\mathrm{S}$, et al. [25]. In elderly individuals, disease resistance is reduced and body ageing is accelerated; thus, it is common to develop chronic diseases. The physical and psychological status of elderly individuals is fragile and slow to recover [26]. The more serious the patient's condition is, the higher the cost of medication becomes. Therefore, the government should increase preventive inputs to improve the health of the people; strengthen the promotion of health concepts; raise awareness of the prevention and control of chronic diseases; strengthen the implementation of early screening for chronic diseases; conduct regular free medical examinations; promote opportunistic screening for chronic diseases such as diabetes, hypertension, and coronary heart disease; establish and improve the health management of chronic diseases; and promote the development of a healthy China. 


Variable
Sex
female
male
Age
0 45 years old
$45 \sim 65$ years old
>65 years old
Medical payment method
OOP
UEMI
URMI
RCMl
Category of major drug
Non-Western Medicine
Western Medicine
Doctor's title
primary
Deputy senior
Senior
Department
Other
Endocrinology and metabolism
General internal medicine
TCM -WM
Cardiovascular
Full implementation of the zero mark-up policy
Before
After

Figure 7: Forest map of coronary heart disease.

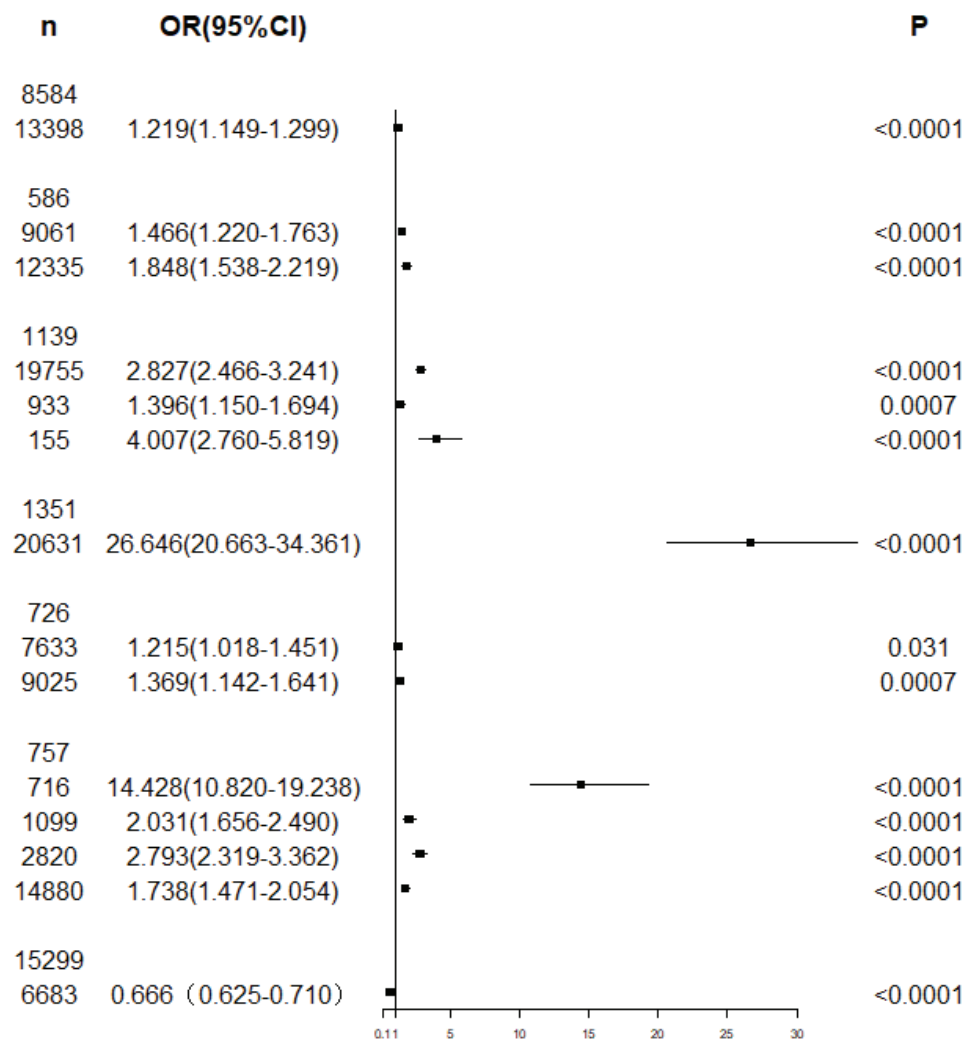

The implementation of the basic medical insurance system aimed to reduce the economic burden of policyholders to a great extent and provide medical protection [27]. According to the current research, there are significant differences in the costs associated with the OOP, UEMI, URMI, and RCMI payment methods. The multivariate logistic regression analysis of the studied chronic diseases showed that there were significant differences in the number of visits among the patients utilizing the different payment modes; the UEMI patients had the highest number of visits. Compared with OOP, UEMI and RCMI were influencing factors of high outpatient drug costs. In addition, costs associated with URMI were different from those associated with OOP for different chronic diseases. For diabetes, there was no difference in outpatient drug costs between the URMI and OOP payment methods. For hypertension, URMI was an influencing factor of low outpatient drug costs compared with OOP, and the OR $(95 \% \mathrm{CI})$ was 0.848 (0.7540.955). For coronary heart disease, URMI was an influencing factor of high outpatient drug costs, and the OR (95\% CI) was 1.396 (1.1501.694). The reasons for the varying results may be mainly from two aspects. First, although chronic diseases such as hypertension, diabetes, and coronary heart disease have been included in the reimbursement program for special disease clinics, the criteria for coverage of certain diseases are strictly limited. For example, hypertension needs to be classified as a high-risk hypertension, which excludes patients with mild hypertension and reduces the compensation coverage of outpatient drug costs. Second, because diabetes and high blood pressure drugs are conventional drugs, residents can easily obtain them from the nearest pharmacy or primary health care institution, thus affecting outpatient drug costs. Min F, et al. [28] suggested that the popularity of medical insurance payment methods has changed the patient ability to pay and reduced the sensitivity of patients to drug costs and treatment costs. To a certain extent, insurance coverage will promote an increase in medical expenses, resulting in the consumption of medical resources and unequal utilization of medical care [29]. This phenomenon is related to the imbalance in China's existing medical insurance system. Moreover, it is also due to the differences in the level, quality and cost burden of different medical insurance services [19]. Therefore, while continuing to implement "full coverage" medical insurance, insurance providers should also pay attention to the control and management of the number of prescription drugs prescribed by doctors to avoid the excessive use of high-priced and imported drugs. In addition, patients should also change their thinking to prevent excessive purchases of drugs due to the high rate of reimbursement by medical insurance. This change can reduce medical resource waste and effectively control the total cost of drugs.

According to the analysis, the cost of outpatient drugs was affected by the doctor's title. In this study, we found that most of the patients with diabetes selected doctors with intermediate, deputy senior, and senior titles, which was an inflation factor for outpatient drug expenses; the associated ORs (95\% CI) were 1.057 (1.021-1.194), 1.046 (1.011-1.084), and 1.441 (1.389-1.495) respectively. However, patients with hypertension and coronary heart disease were more likely to select doctors with deputy and senior titles. Compared with the other doctor titles, deputy senior and senior titles were influencing factors for increased drug expenses. Four reasons might lead to the association between outpatient drug expenses and doctors' titles. First, access to clinical resources and medical technologies are the main consideration 
when patients choose doctors. This is also why most patients tend to choose highly qualified directors and specialists. High-ranking doctors have more experience and more drug treatment options than lowranking doctors, and they prefer to prescribe expensive drugs. Second, in the analysis of doctor titles associated with diabetes mellitus costs, the intermediate title was also an influencing factor for increased drug costs. This may be related to the number of intermediate-level doctors. Compared with doctors who treat hypertension and coronary heart disease, most doctors who treating diabetes have intermediate-level titles. The reason might be that compared with some primary-level doctors, their clinical experience is extensive and the medication they prescribe is more efficient. Intermediate-level doctors tend to use drugs that are quick, effective, and expensive. Therefore, the use of intermediate-level doctors may also be a reason for the increase in drug costs. Third, with "comprehensive" coverage by medical insurance, payment responsibility and economic affordability have been improved, and patients can access high-quality health services, which indirectly affects doctors' diagnosis and treatment behaviours and promotes the use of relatively expensive drugs. Fourth, more experience doctors receive more severe cases, so patients have higher drug costs. Thus, to control outpatient drug costs, standardized management, standardized clinical drug use, increased prescription management, and strengthened medical ethics need to be implemented. Medical institutions are responsible for strictly implementing the hierarchical diagnosis system [30]; guiding the rational flow of patients who seek medical treatment; gradually improving and implementing the graded diagnosis and treatment models of primary diagnosis, twoway referrals, and short-term and long-term treatment; standardizing clinical pathways for chronic diseases; constructing multi-centre and multi-state clinical pathways; and reducing medical costs and health care resource waste. Hospitals need to strengthen the construction of grassroots medical institutions and train their doctors in the diagnosis and treatment of chronic diseases. In addition, for patients, hospitals need to establish rational medication concepts, utilize appropriate symptomatic drugs, and avoid the excessive prescription of highpriced drugs and imported drugs.

Since the implementation of the zero mark-up policy in Chongqing, there have been some changes in the outpatient drug costs for diabetes, hypertension and coronary heart disease. The analysis showed that there was a significant difference in the drug expenses before and after the full implementation of the zero mark-up policy (diabetes OR: 0.797, 95\% CI: 0.781-0.814, P<0.0001; hypertension OR: 0.753, 95\% CI: 0.732-0.774, $\mathrm{P}<0.0001$; coronary heart disease OR: $0.666,95 \% \mathrm{CI}$ : $0.625-0.710, \mathrm{P}<0.0001)$. The full implementation of the zero mark-up policy was an influencing factor for outpatient drug expenses. Since the implementation of this policy on September 9, 2017, the expenses of medications for patients with NCDs has been reduced. The cost of diabetes drugs decreased from $604.65(344.90,1012.63)$ Yuan to $548.35(317.29,901.15)$ Yuan; the cost of drugs in hypertension clinic decreased from $484.79(253.60,825.50)$ Yuan to $435.78(248.22,735.63)$ Yuan; the cost of outpatient drugs for coronary heart disease decreased from $714.16(420.06,1028.20)$ Yuan to $621.02(369.58,935.51)$ Yuan. The full implementation of the zero mark-up policy effectively controls outpatient drug expenses, prevents the situation of "supplementing hospital expenses with medication revenue", standardizes doctors' prescribing behaviours, prevents doctors from prescribing expensive drugs and obtaining rebates, encourages hospitals to adjust the cost structure, improves patients' medical experiences, and further enhances the value of medical services [31,32]. However, the zero mark-up policy also has some negative effects. The policy has led to increased operating pressure, reduced enthusiasm of doctors, and overworked medical staff in hospitals. At the same time, because the retail price of drugs in hospitals is consistent with the drug prices available to patients, some patients are willing to buy drugs from local pharmacies, which lead to the accumulation of drug stocks in hospitals [33]. Therefore, the government should actively integrate resources, improve relevant policies, increase financial subsidies, further promote the medical insurance system, improve the drug zero mark-up policy, improve the value of medical services, and implement a reasonable compensation mechanism for doctors. Hospitals should change their management strategies, implement the close oversight of industrial structures, improve medical quality and standardize diagnosis and treatment behaviours $[34,35]$.

\section{Conclusion}

This study showed that the outpatient drug expenses for diabetes, hypertension, coronary heart disease and other chronic diseases were affected by many factors, and there were obvious differences in the drug costs among the different chronic diseases. The economic burdens of drug costs were $687.53(404.76,1001.62)$ Yuan for coronary atherosclerotic heart disease, $582.50(340.76,973.53)$ Yuan for diabetes mellitus and $473.30(252.18,804.68)$ Yuan for hypertension. The economic burden of coronary heart disease was the highest and most serious.

There were some limitations in the present study. Firstly, the generalizability of the results is a concern, as this study was limited to a tertiary, a hospital in Chongqing; these patients cannot be considered representative of all patients with NCDs in Chongqing. In the future, the scope of research will be expanded, and multi-centre and multicoverage research will be carried out. Secondly, because of the few variables in the study, the considered factors were limited, and we were not able to conduct a comprehensive analysis. The thirdly limitation is that we didn't know the actual insurance reimbursement range and expense ratio for most subjects in this study. Fourthly, we were not looking for additional social burden, there was not enough information that was collected that included family incomes, jobs, education levels, in lost productivity, use of other type of healthcare services. In the future, we need to study socio-economic and drug economy factors and carry out in-depth policy improvement and health economics evaluations [36]. Moreover, China needs to strengthen the analysis of hospital outpatient revenues, scientifically and accurately analyse the rational allocation of medical and health resources to promote hospital outpatient services and provide high-quality and high-level medical and health services.

\section{Acknowledgement}

We would like to thank the Humanities and Social Science Project of Chongqing Municipal Education Commission for the support of this research. We would also like to thank all the teachers and students who made invaluable comments and suggestions for this paper.

\section{Conflicts of Interest}

The authors declare no conflicts of interest.

\section{References}

1. Xu Y, Garrib A, Zhou Z, Wang D, Gao J, et al. (2019) New Health Care Reform and Impoverishment among Chronic Households in China: A Random-Intercept Approach. Int J Environ Res Public Health 26: pii: E1074.

2. World Health Organization (2018) Noncommunicable diseases. WHO, Geneva, Switzerland. 
3. Theme Filha MM, de Souza Junior PR, Damacena GN, Szwarcwald $C L$ (2015) Prevalence of chronic non-communicable diseases and association with self-rated health: National Health Survey, 2013. Rev Bras Epidemiol 18: 83-96.

4. Nulu S (2017) Neglected chronic disease: The WHO framework on non-communicable diseases and implications for the global poor. Glob Public Health 12: 396-415.

5. World Health Organization (2014) Global Status Report on Noncommunicable Diseases. WHO, Geneva, Switzerland.

6. Cause of Death by Non-Communicable Diseases. The World Bank, USA.

7. Hajat C, Stein E (2018) The global burden of multiple chronic conditions: A narrative review. Prev Med Rep 12: 284-293.

8. Patrick Richard, Regine Walker, Pierre Alexandre (2018) The burden of out of pocket costs and medical debt faced by households with chronic health conditions in the United States. PLoS One 13: e0199598.

9. Rahman MM, Gilmour S, Saito E, Sultana P, Shibuya K (2013) Healthrelated financial catastrophe, inequality and chronic illness in Bangladesh. PLoS One 8: e56873.

10. Kien VD, Minh HV, Ngoc NB, Phuong TB, Ngan TT, et al. (2017) Inequalities in Household Catastrophic Health Expenditure and Impoverishment Associated With Noncommunicable Diseases in Chi Linh, Hai Duong, Vietnam. Asia Pac J Public Health 29: 35S-44S.

11. Yip WC, Hsiao WC, Chen W, Hu S, Ma J, et al. (2012) Early appraisal of China's huge and complex health-care reforms. Lancet 379: 833842

12. Li X, Xu Z, Ji L, Guo L, Liu J, et al. (2019) Direct medical costs for patients with type 2 diabetes in 16 tertiary hospitals in urban China: A multicenter prospective cohort study. J Diabetes Investig 10: 539551.

13. Liu GG, Vortherms SA, Hong X (2017) China's Health Reform Update. Annu Rev Public Health 38: 431-448.

14. Fang K, Shia B, Ma S (2012) Health insurance coverage and impact: a survey in three cities in China. PLoS One 7: e39157.

15. Luo C, Liu Y (2016) Drug Price Formation Mechanism and Governance. Human Resource Management 201-202.

16. Yi H, Miller G, Zhang L, Li S, Rozelle S (2015) Intended and unintended consequences of China's zero markup drug policy. Health Aff (Millwood) 34: 1391-1398.

17. Robles S, Anderson GF (2011) Continuity of care and its effect on prescription drug use among Medicare beneficiaries with hypertension. Med Care 49: 516-521.

18. Cortaredona S, Ventelou B (2017) The extra cost of comorbidity: multiple illnesses and the economic burden of non-communicable diseases. BMC Medicine 15: 216

19. Jinglin D, Ye W, Mingsheng C, Zhonghua W (2019) Empirical Analysis of the Actual Reimbursement of Basic Medical Insurance of Outpatient Services and Influencing Factors. Chinese Hospital Management 39: 50-52

20. Li Q, Yu Y, Kong Q, et al. (2019) Study on Outpatient Visit Volume Prediction and Lean Management in a Psychiatric Hospital. Hospital Management Forum 36: 34-37.
21. Yin $X, X u$ Y, Man X, Liu L, Jiang $Y$, et al. (2019) Direct costs of both inpatient and outpatient care for all type cancers: The evidence from Beijing, China. Cancer Med 8: 3250-3260.

22. Wang Z, Li X, Chen M (2015) Catastrophic health expenditures and its inequality in elderly households with chronic disease patients in China. Int J Equity Health 14: 8.

23. Wang Z, Li X, Chen M, Si L (2018) Social health insurance, healthcare utilization, and costs in middle-aged and elderly communitydwelling adults in China. Int J Equity Health 17: 17.

24. Lu WH, Lee WJ, Chen LK, Hsiao FY (2016) Comparisons of annual health care utilization, drug consumption, and medical expenditure between the elderly and general population in Taiwan. J Clin Gerontol Geriatr 7: 44-47.

25. Wang S, Petzold M, Cao J, Zhang Y, Wang W (2015) Direct medica costs of hospitalizations for cardiovascular diseases in Shanghai, China: trends and projections. Medicine (Baltimore) 94: e837.

26. Mao-Juan H, Min P, Ying-Ming W, Mei Z, Lian-Zi Z (2017) Analysis on hospitalization cost compositions and influence factors of elderly patients with chronic diseases in Sichuan province. Soft Sci of Health 31: 15-19.

27. Wang S, Liu L, Li L, Liu J (2014) Comparison of Chinese inpatients with different types of medical insurance before and after the 2009 healthcare reform. BMC Health Serv Res 14: 443.

28. Min F, Shaolong WU (2017) Did "New Medical and Healthcare Systems Reform" Bring Medical Expenses Down: Based on the Trace Data of CHARLS of Gansu and Zhejiang. J Beijing Adm Inst 18-27.

29. Hu H, Sawhney M, Shi L, Duan S, Yu Y, et al. (2015) A systematic review of the direct economic burden of type 2 diabetes in china. Diabetes Ther 6: 7-16.

30. Niu W, Huang J, Xing Z, Chen J (2019) Knowledge Spillovers of Medical Big Data Under Hierarchical Medical System and Patients' Medical Treatment Decisions. IEEE Access 7: 55770-55779.

31. Lian Y (2015) Analyzing the Effects of Implement the Zero-markup Drug Policy in County-level Public Hospital in Sichuan Province. Chinese Health Service Manag 32: 429-433.

32. Ping J, Heng W, Niannian L, Hongyan Y, Cunhui W (2018) Influence and countermeasures of eliminating drug price addition on operating mechanism of public hospitals. Journal of Nanjing Medical University (Social Sciences) 18: 372-376.

33. Lihang L, Feiyang $T$ (2019) Predicament of Performance Reform of County-Level Public Hospitals Under the Background of Drug ZeroAddition. Chinese Hospital Management 39: 10-11.

34. Shu-mei L, Wen-hua H, Jin-yu C, Xiamen maternity and child health care hospital (2016) The Impact and Suggestion for Implementing Drug Price Zero-addition Policy on Economy Operation of the Specialized Hospitals. Chinese Health Economic 23-25.

35. Li Q, Chen F, Yang M, Lu L, Pan J, et al. (2018) The Effect of China's National Essential Medicine Policy on Health Expenses: Evidence From a National Study. Inquiry 55: 46958018787057.

36. Yang C, Huang Z, Sun K, Hu Y, Bao X (2018) Comparing the Economic Burden of Type 2 Diabetes Mellitus Patients with and without Medical Insurance: A Cross-Sectional Study in China. Med Sci Monit 24: 3098-3102. 